\title{
IMPLEMENTATION STRATEGY TO MINIMISE THE SPECKLE NOISE FROM POLARIMETRIC SAR DATA
}

\section{RAMANA RAO KV ${ }^{1}$, NAGENDRABABU MAHAPATRUNI ${ }^{2}$, VELANGINISARAT $P^{3}$ \& KALLEMPUDI VAHINI $^{4}$}

${ }^{1}$ Associate Professor, Department of Electronics and Communications Engineering, Vignan's Institute of Engineering for Women, Visakhapatnam, Andhra Pradesh, India

${ }^{2}$ Associate Professor, Department of Mechanical Engineering, Vignan's Institute of Engineering for Women, Visakhapatnam, Andhra Pradesh, India

${ }^{3}$ Assistant Professor, Department of Electrical and Electronics Engineering, Vignan's Institute of Engineering for Women, Visakhapatnam, Andhra Pradesh, India

${ }^{4}$ Assistant Professor, Department of Mechanical Engineering, Vignan's Institute of Engineering for Women, Visakhapatnam, Andhra Pradesh, India

\begin{abstract}
Polarimetric Synthetic Aperture Radar (PolSAR) is an advanced imaging radar system which emits electromagnetic pulses and receives echo signals reflected by ground objects in order to obtain scattering characteristics of various objects on the earth surface. This paper helps in the study of scattering of corona molecules through splitting or sneezing. The data collected by a PolSAR is mostly affected by speckle noise. This speckle noise occurs due to the interaction between coherent signals returning from many scatters present on the earth surface. The presence of this speckle noise causes difficulties in interpretation and analysis during image processing. Hence the suppression of this speckle noise in SAR data is important for extraction of meaningful information for PolSAR images. This paper proposes minimization of speckle noise from PolSAR data by using a set of speckle filters such as boxcar, median, refined Lee and IDAN. The performance can be measured by calculating certain parameters such as mean square error(MSE), peak signal to noise ratio(PSNR), standard deviation to mean ratio (SD/M), edge preservation index (EPI) and equivalent number of looks(ENL).

KEYWORDS: Polarimetric Synthetic Aperture Radar(Polsar), Speckle Noise, Speckle Filters, Edge Preservation Index
\end{abstract}

Received: Jun 06, 2020; Accepted: Jun 26, 2020; Published: Jul 29, 2020; Paper Id.: IJMPERDJUN2020520

\section{INTRODUCTION}

Polarimetric Synthetic Aperture Radar (SAR) technology plays major role for remote sensing applications by monitoring the natural features on earth surface and by analysing the periodical changes as and when required. In order to obtain high spatial resolution in terms of image reflectivity properties, initially systems employed a single channel configuration. The advanced multi-dimensional SAR system provides the maximum amount of information present on earth surface. Based on the polarization diversity, Polarimetric SAR (PolSAR) systems are emerged as main system configuration (Huang, et al.) (Thankachan, Sethunadh, \& Ameer, 2019).PolSAR data depends on scattering properties of image and geometry of scatters present on the earth surface.

After the establishment of different space born sensors with different polarimetric capabilities, the focus on PolSAR data has been increased a lot. For every pixel, PolSAR system measures the scattering matrix. For the deterministic scatters, scattering matrix calculates the complete scattering processing being considered (Khare $\&$ Kaushik, 2019). But in case of distributed scatters and because of coherent nature of SAR system,the scattering 
matrix is random since the scattering process is complexin this case.The nature of SAR data is referred to as speckle noise(Shaik Mahaboob Basha \& Jinaga, 2020) (Liu, Lu, Shen, Xu, \& Xu, Multiplicative Noise Removal: Nonlocal LowRank Model and Its Proximal Alternating Reweighted Minimization Algorithm, 2020).Speckle noise reduction has to be performed without losing the actual information content and preserving the edges and features (Mahapatruni, Gopi Krishna, Gandi, \& Kuriti, 2020) (Liu, Yang, Yang, Topics, \& 2019).

\section{DESIGN METHODOLOGY}

Various Methods are Proposed to Reduce Speckle Noise by Applying Several Types of Filters. This Paper has been Organised as Follows:

- PolSAR data speckle filters

- Boxcar filter

- Median filter

- $\quad$ Refined lee filter

\subsection{PolSAR Data Speckle Filters}

Filtering is a technique used to remove unwanted information from an image, so it provides better performance during image processing (Zhang, Hu, Water, \& 2020).Speckle filters are mainly used to remove noise in uniform area without change in features and provide good appearance. The main objective of the present work is to compare different PolSAR speckle noise filters to find the ability of different approaches. This section describes short definition and mathematical formulae of various speckle filters (Chen B. , Zou, Chen, \& Zhang, 2019) (Basha, Computing, \& 2020).

\subsection{Boxcar Filter}

Boxcar filter is simple averaging filter that replaces the pixel in square kernel by the mean value of kernel pixels. This filter has good performance in reducing the speckle noise in homogeneous area. Mathematically represented as:

$$
x(i, j)=<y\left(i, j_{N}\right)>=\left(\frac{1}{N^{2}}\right) \sum_{p=\frac{-N}{2}}^{p=\frac{N}{2}} \sum_{q=\frac{-N}{2}}^{q=\frac{N}{2}} Y_{i+p, j+q}
$$

Where $i$ and $j$ represents the number of rows and columns, $X(i, j)$ represents the estimated pixel value constructed by computing the sample mean over each pixel neighbourhood and $y(i, j)$ represent original pixel.

\subsection{Median Filter}

This filter considered as spatial nonlinear filter. For reducing pulse or speckle noise, this filter works by calculating the median value of its neighbours in the window can be implemented based on the formulae below:

$$
\mathrm{f}^{\wedge}(\mathrm{x}, \mathrm{y})=\operatorname{median}_{(\mathrm{s}, \mathrm{t}) \in \mathrm{s}_{\mathrm{xy}}(\mathrm{x}, \mathrm{y})}\{\mathrm{g}(\mathrm{s}, \mathrm{t})\}
$$


Where $g(s, t)$ gives the original image, $S_{x y}$ represent the set of coordinates in rectangular image windows. This filter is capable to remove impulse or short noise, but it is not well suited for speckle noise.

\subsection{Refined Lee Filter}

The lee filters compute a linear combination of the centre pixel intensity in filter window with an average intensity of the window for removing speckle noise. These filters based on the minimum mean square error(MMSE) and speckle noise and the image is produced based on the following equation.

$$
\mathrm{R}^{\wedge}(\mathrm{t})=\overline{\mathrm{I}}(\mathrm{t})+\mathrm{W}(\mathrm{t})(\mathrm{I}(\mathrm{t})-\overline{\mathrm{I}}(\mathrm{t}))
$$

Where $R^{\wedge}(t)$ is the image value after being filtered and it is also estimated value of $R(t), \bar{I}(t)$ is the mean value of $I(t)$ and the weighting function $\mathrm{W}(\mathrm{t})$ is given by

$$
\mathrm{W}(\mathrm{t})=1-\mathrm{CV} / \mathrm{CI}
$$

Where $\mathrm{CV}$ is the variance coefficient of noise affected image with standard deviation and CI is the variance coefficient of noise free of local image with standard deviation.

\section{PoISAR DATA FILTER EVALUATION PROCEDURE}

In particular, a good SAR de speckling technique should have special characteristics such as speckle reduction in homogeneous area and feature preservation and also radiometric preservation. The following is the process to evaluate some parameters like mean square error MSE, peak signal to noise ratio (PSNR), edge preservation index (EPI), equivalent number of looks $(\mathrm{ENL})$,Standard deviation to mean SD/M .

\subsection{Mean Square Error}

Mean square error is parameter used to find the difference of original image and de noised image. The values indicate the difference between the original and filtered image. Mean square error is calculated as follows

$$
\operatorname{MSE}(\mathrm{A}, \mathrm{B})=\operatorname{Mean}\left[(\mathrm{A}-\mathrm{B})^{2}\right]
$$

Where A and B represent Original and filtered images. The larger the value of MSE represent original and filtered images is not similar and smaller values of MSE represent the better quality of image.

\subsection{Peak Signal to Noise Ratio}

This parameter can be defined as ratio between maximum samples allowed by original data to that of noise affected data. Mathematically PSNR can be represented as 


$$
\mathrm{PSNR}=10 * \log _{10}\left[\frac{\mathrm{X}_{\mathrm{p} 1}}{\mathrm{MSE}}\right]
$$

Where,

$$
\mathrm{X}_{\mathrm{p} 1}=\text { maximum samples of }(\mathrm{A}) \& \mathrm{X}_{\mathrm{p} 2}=\text { maximum samples of }(\mathrm{B})
$$

\subsection{Standard Deviation to the Mean Ratio}

This is also known as co- efficient of variance $(\mathrm{CV})$ and this parameter is ratio of standard deviation to the mean. This ratio is commonly used to measure the reduction of speckle noise(Thankachan, Sethunadh, \& Ameer, 2019).Better speckle noise reduction gives less $\mathrm{SD} / \mathrm{M}$ value. Mathematically $\mathrm{SD} / \mathrm{M}$ can be represented as

$$
\mathrm{SD} / \mathrm{M}=\mathrm{CV}=\beta=\frac{\sqrt{\operatorname{var}(\mathrm{B})}}{\sqrt{\operatorname{mean}(\mathrm{B})}}
$$

Where $\beta$ represents Filtered data.

\subsection{Edge Preservation Index}

Edge preservation index is defined as

$$
\mathrm{EPI}=\frac{\mathrm{X}_{\mathrm{p} 21}-\mathrm{X}_{\mathrm{P} 22}}{\mathrm{X}_{\mathrm{p} 11}-\mathrm{X}_{\mathrm{p} 12}}
$$

Where $\mathrm{X}_{\mathrm{p} 21} \mathrm{X}_{\mathrm{p} 22} \mathrm{X}_{\mathrm{p} 11} \mathrm{X}_{\mathrm{p} 12} \mathrm{Xp}_{21}$ and $\mathrm{Xp}_{22}$ are the values of filtered and original images observed on both sides of edges respectively. Better edge ability is indicated by larger EPI values.

\subsection{Equivalent Number of Looks}

Equivalent number of looks for intensity image is defined as

$$
\mathrm{ENL}=\frac{1}{\beta^{2}}
$$

Equivalent number of looks for amplitude image is defined as

$$
\mathrm{ENL}=\left(\frac{0.522}{\beta}\right)^{2}
$$

Where $\beta$ indicates standard deviation to the mean ratio.

\section{RESULTS AND EVALUATIONS}

\subsection{Filtered Images}

The polarimetric synthetic aperture radar data has been processed in SNAP software in two stages as TYPE 1 and TYPE 2 
and the filtered data is exported as geo tiff format in order to provide input to the MATLAB software tool.

\subsection{Raw Data Filtered Output}

The raw data is downloaded from the polSAR sensors ENVISAT and SENTINEL and preprocessed with SNAP software tools and are analyzed in MATLAB. It is shown in Figure 1.

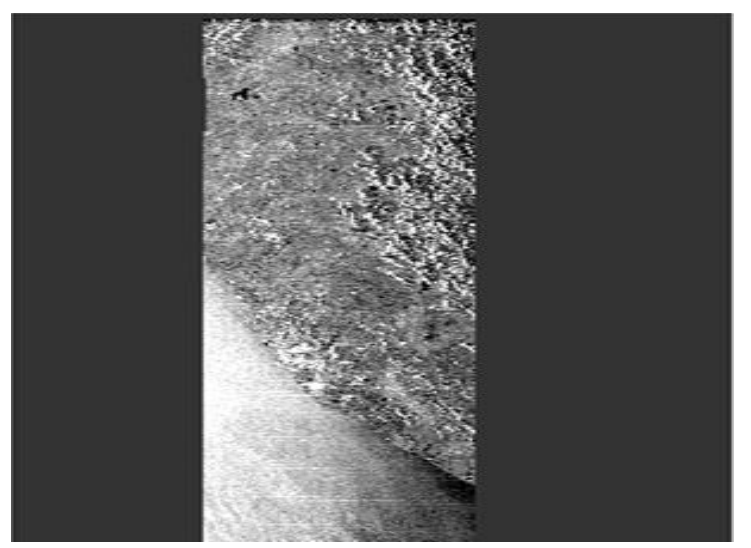

Figure 1: Raw Data with VV Polarization.

\subsection{Boxcar Filtered Output}

The boxcar filter is applied for $\mathrm{VV}$ and $\mathrm{HH}$ polarization modes of various windows size such as 3 and 5 and the respective image are shown in the following figures (Figure $2-7$ ).

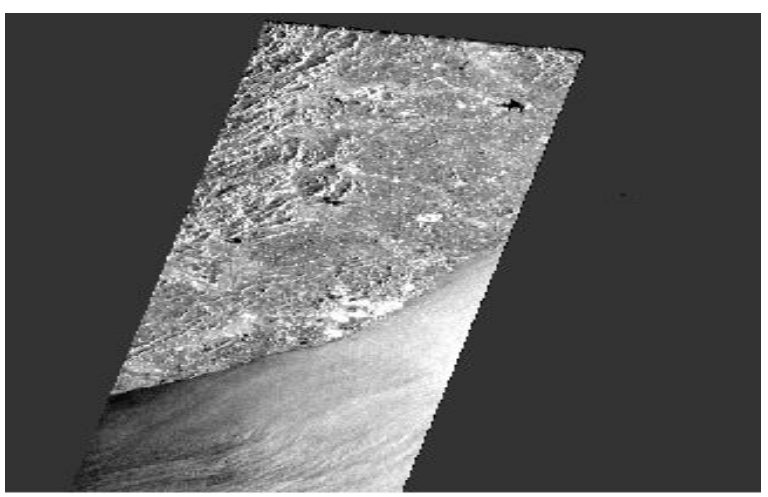

Figure 2: Boxcar 3*3 Filtered Output for VV Polarization.

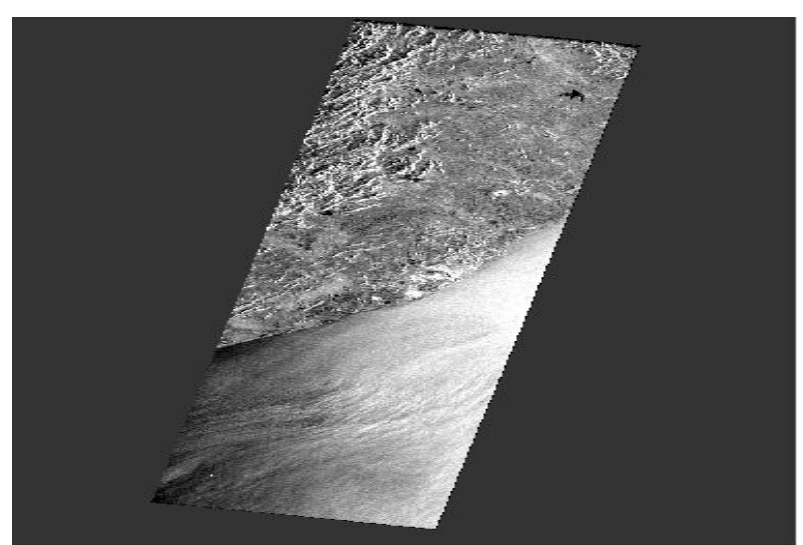

Figure 3: Boxcar 3*3 Filtered Output for HH Polarization. 


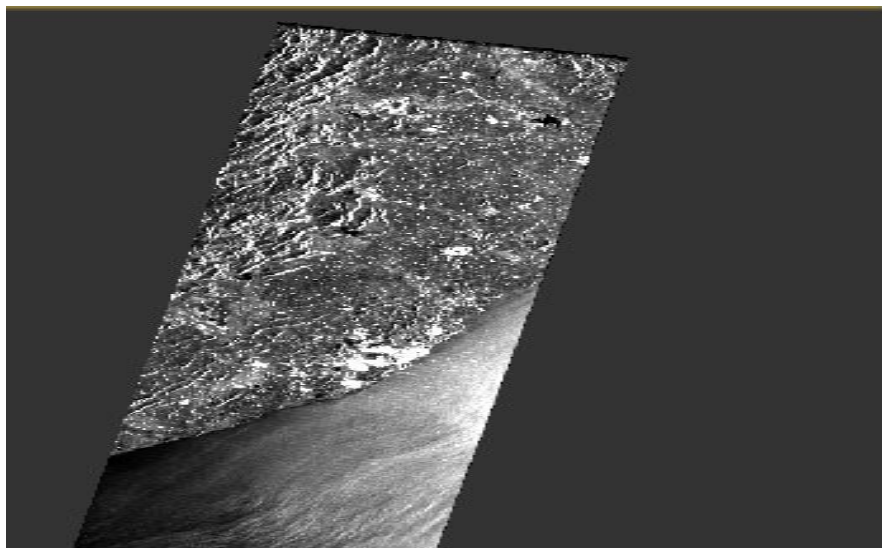

Figure 4: Boxcar 5*5 Filtered Output for VV Polarization.

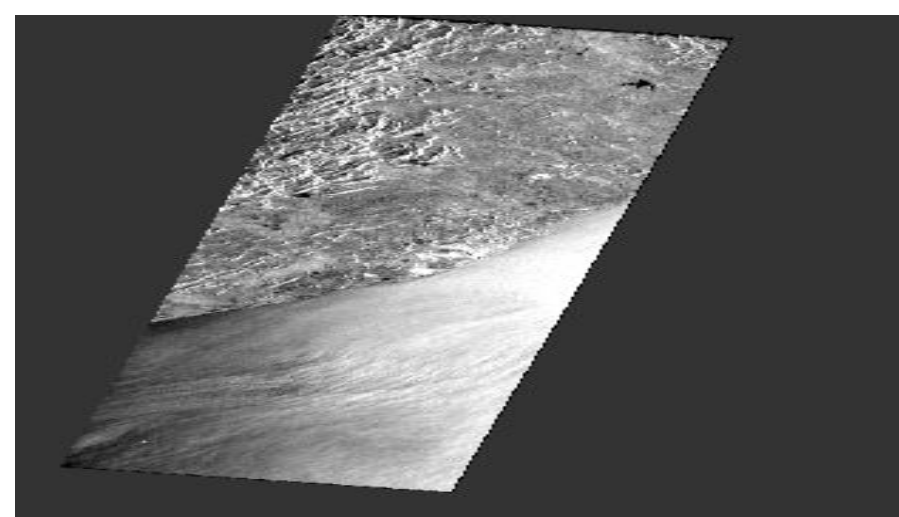

Figure 5: Boxcar 5*5 Filtered Output for HH Polarization.

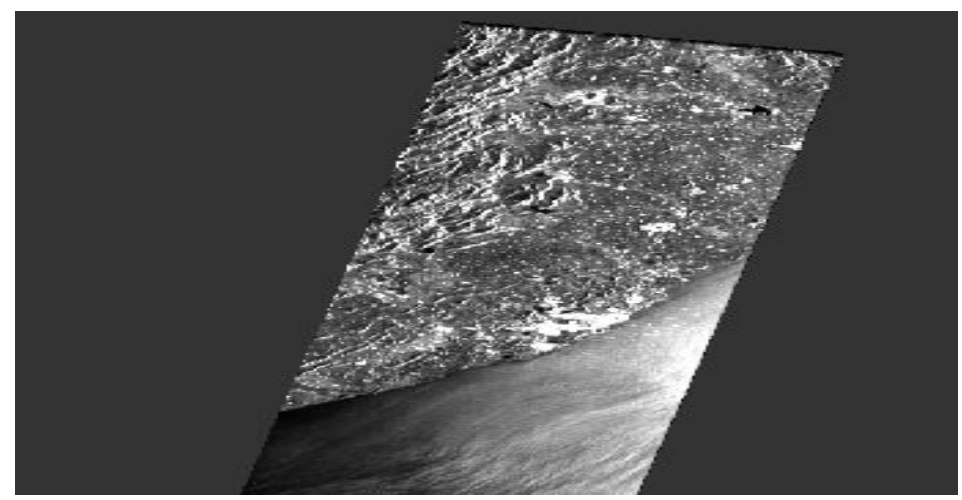

Figure 6: Boxcar7*7 Filtered Output for VV Polarization.

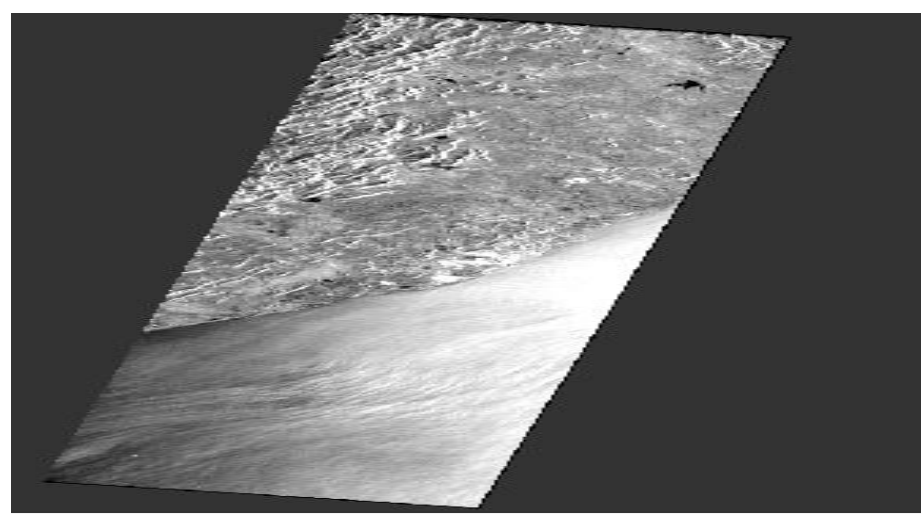

Figure 7: Boxcar 7*7 Filtered Output for HH Polarization. 


\subsection{Median Filtered Output}

The median filter is applied to the type 1 data and VV and HH polarization modes are obtained and illustrated from Figure 813.

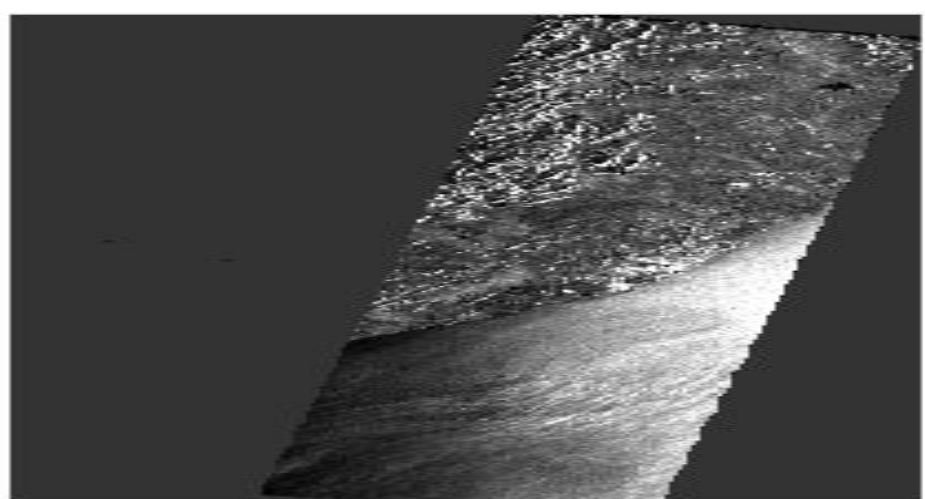

Figure 8: Median 3*3 Filtered Outputs for VV Polarization.

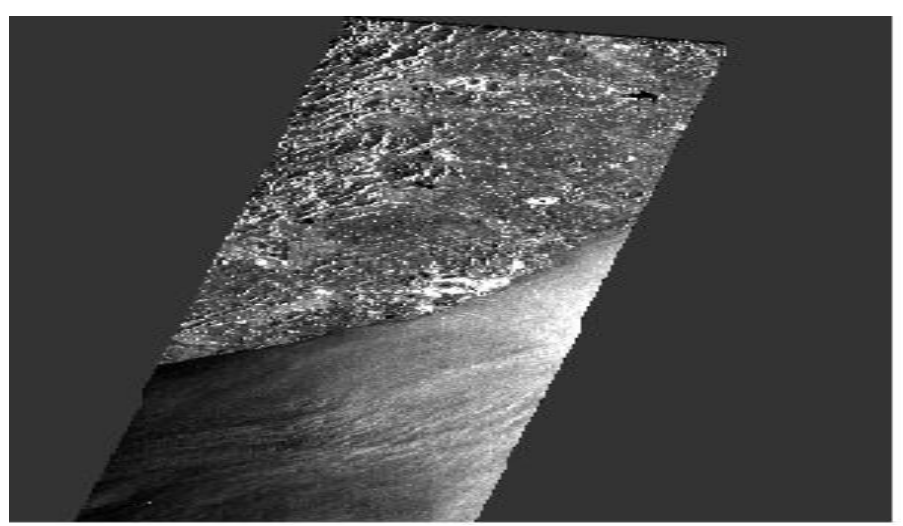

Figure 9: Median 3*3 Filtered Outputs for HH Polarization.

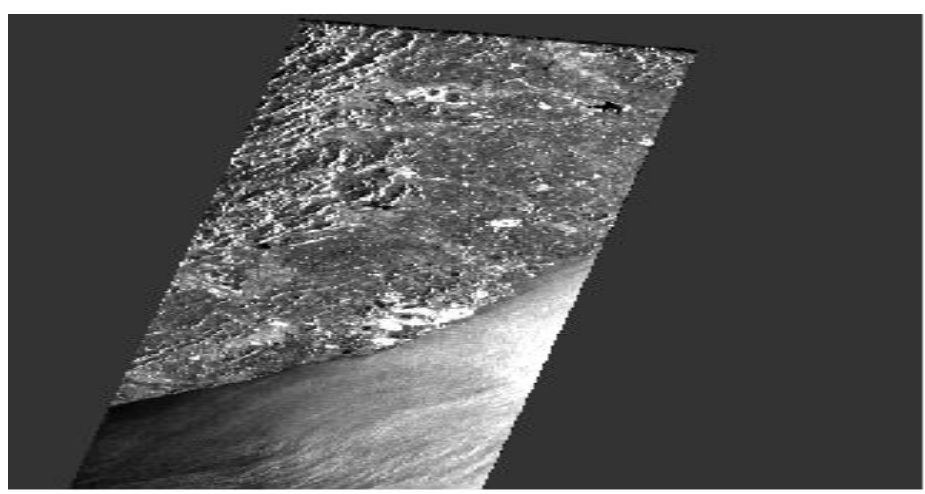

Figure 10: Median 5*5 Filtered Outputs for VV Polarization. 


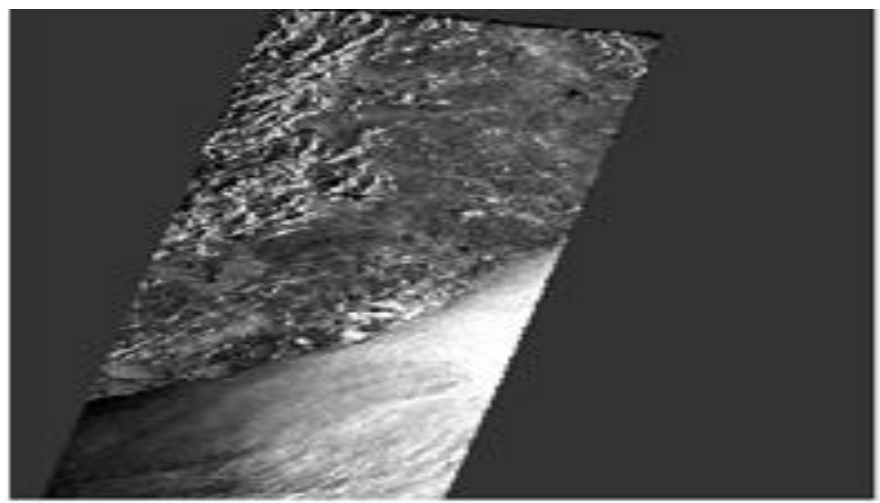

Figure 11: Median 5*5 Filtered Outputs for HH Polarization.

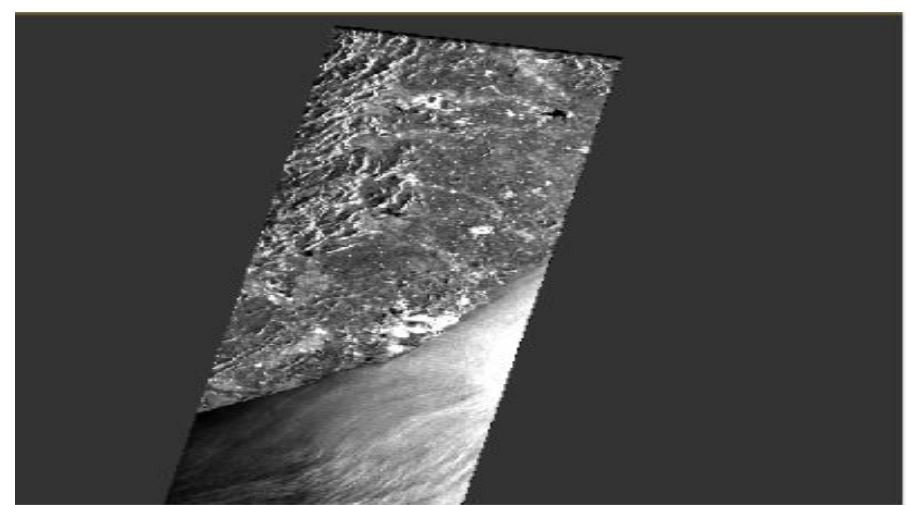

Figure 12: Median 7*7 Filter Output for VV Polarization.

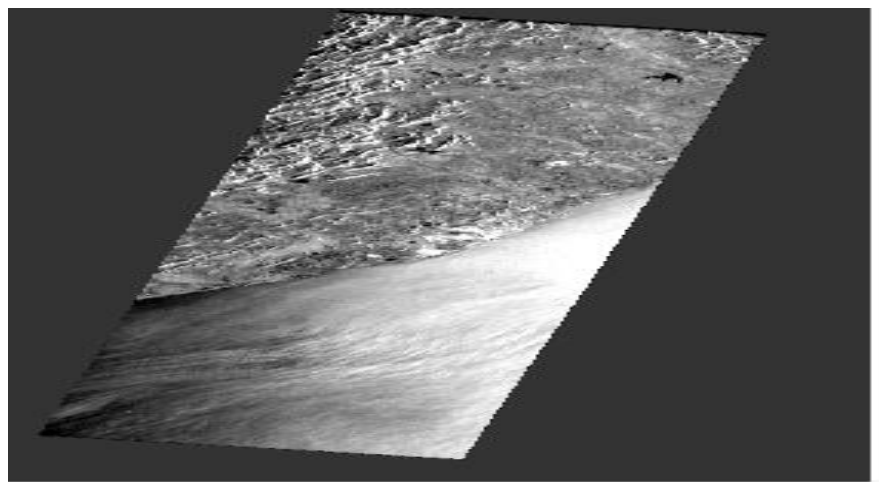

Figure 13: Median 7*7 Filtered Outputs for HH Polarization.

\subsection{Refined Lee Filter Output}

Refined lee filtered outputs are observed for both VV and HH modes of polarization and are illustrated in Figure 14-15.

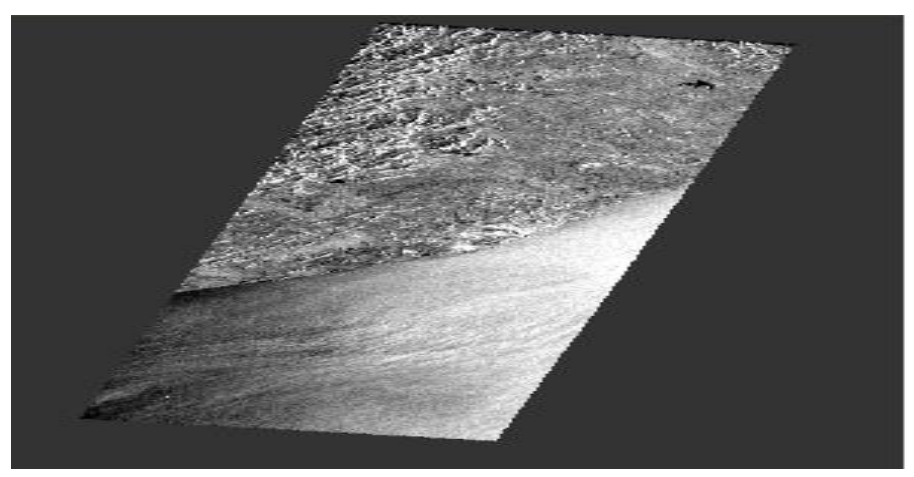


Figure 14: Refined Lee Filter Output for VV Polarization

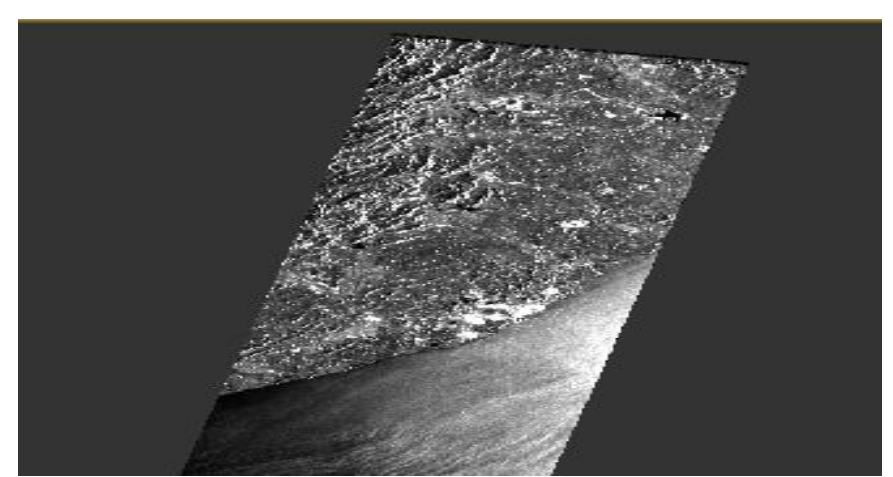

Figure 15: Refined Lee Filter Output for HH Polarization

\subsection{IDAN FILTERED OUTPUTS}

The IDAN Filter is applied on both VV and HH modes of polarization and are illustrated in Figure 16 \& Figure 17.

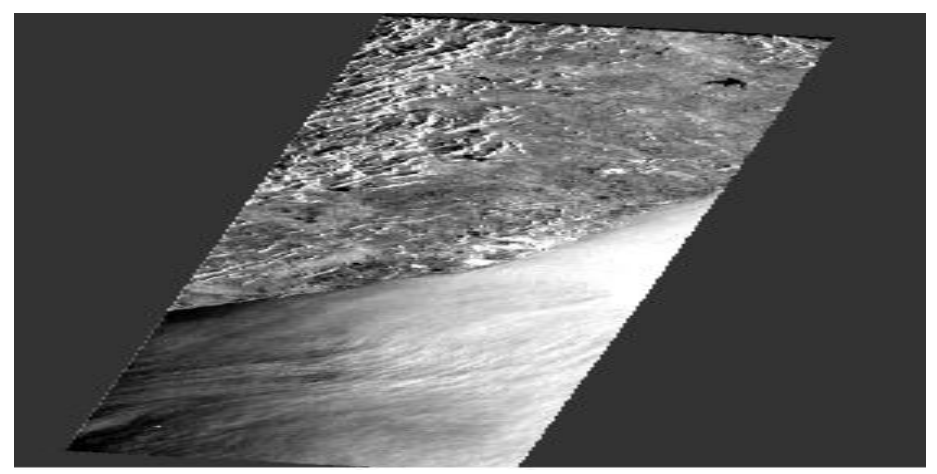

Figure 16: Basic IDAN Filter Output for VV Polarization

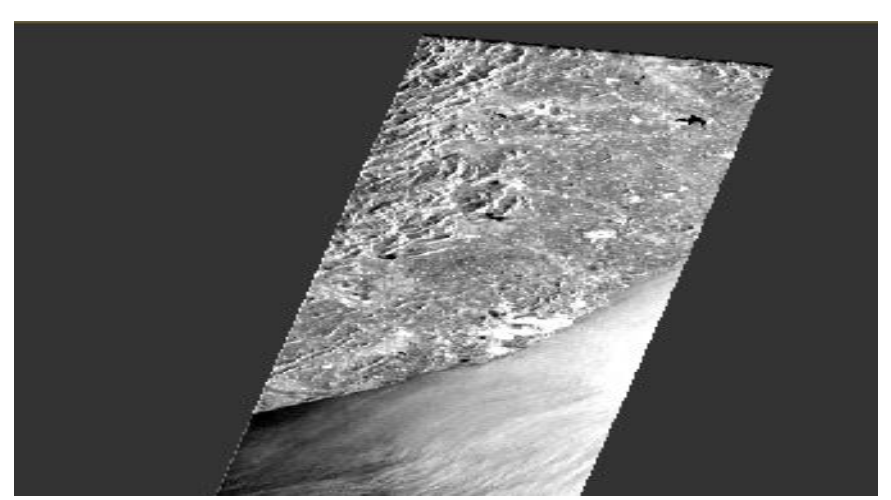

Figure 17: Basic IDAN Filter Output for HH Polarization

\section{RESULTS}

The filtered data is exported in the form of Geo tiff format and is given as input to the MATLAB software tool. The respective values are listed in Table 1.

Table 1: Parameters Calculation of Various Filters

\begin{tabular}{|c|c|c|c|c|c|c|}
\hline FILTER & MSE & PSNR & SD/M & EPI & ENL(A) & ENL(I) \\
\hline Boxcar3 & 89.9176 & 25.1744 & 0.2435 & 1.4262 & 16.8691 & 4.5966 \\
\hline Boxcar5 & 89.7388 & 20.733 & 0.1968 & 1.074 & 25.8249 & 7.0368 \\
\hline Boxcar7 & 90.139 & 16.939 & $\mathbf{0 . 1 7 8 7}$ & 1.3716 & $\mathbf{3 1 . 3 2 6 7}$ & $\mathbf{8 . 5 3 6}$ \\
\hline
\end{tabular}




\begin{tabular}{|c|c|c|c|c|c|c|}
\hline Median3 & 89.9794 & 26.911 & 0.2512 & $\mathbf{1 . 7 9 3 2}$ & 15.8448 & 4.3975 \\
\hline Median5 & 90.4559 & 21.8838 & 0.1978 & 1.2262 & 25.5509 & 6.9622 \\
\hline Median7 & 91.3703 & 19.4679 & 0.2267 & 1.1786 & 19.4521 & 5.3004 \\
\hline Ref. Lee & 90.1604 & 25.934 & 0.2098 & 1.4327 & 22.7289 & 6.1933 \\
\hline IDAN & $\mathbf{8 6 . 9 1 4 7}$ & $\mathbf{2 7 . 1 8 8}$ & 0.339 & 0.913 & 8.7016 & 2.371 \\
\hline
\end{tabular}

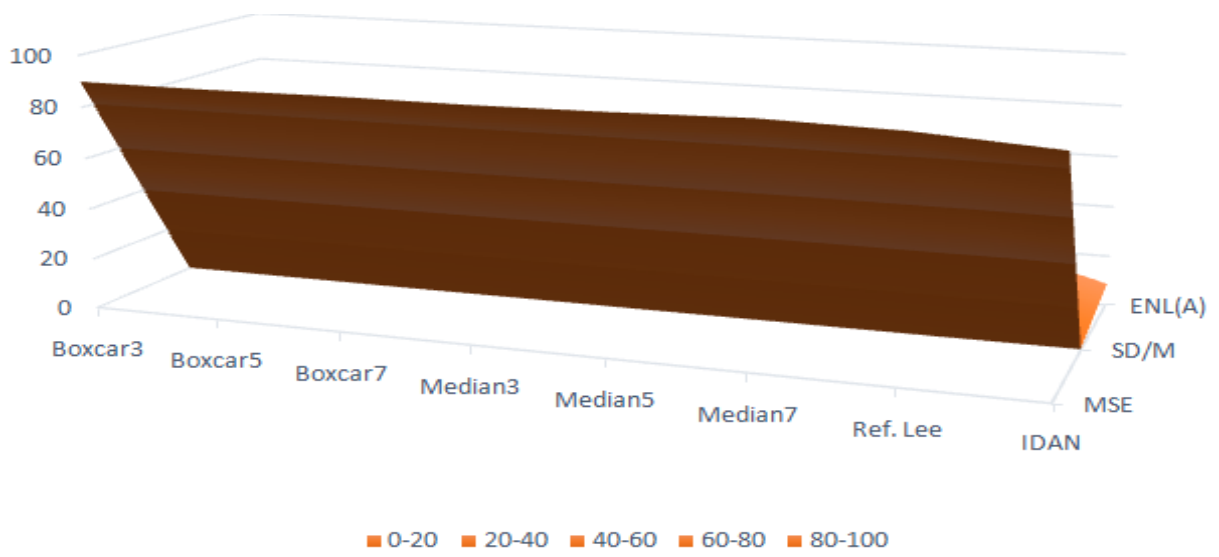

Figure 18: Surface Plot Illustrating Parameter Evaluation with Various Filters.

\section{CONCLUSIONS}

After evaluation and calculation of parameters from different filters such as Boxcar, Median, Refined Lee it is observed that Boxcar filter provides better SD/M ratio and equivalent number of looks for both amplitude and intensity images. Median filter provides better edge preservation index whereas IDAN filter provides better Mean Square Error and Peak signal to Noise ratio. Hence the minimization of speckle noise has been achieved to a greater extent from PolSAR data.

\section{REFERENCES}

1. Basha, S., Computing, B.-I., \& 2020, u. (n.d.). Robust Denoising Technique for Ultrasound Images Based on Weighted Nuclear Norm Minimization. Springer.

2. Chen, B., Zou, J., Chen, W., \& Zhang, M. (2019, 9 1). A novel energy functional minimization model for speckle noise removal. Optoelectronics Letters, 15(5), 386390.

3. Chen, B., Zou, J., Chen, W., Letters, M.-O., \& 2019, u. (n.d.). A novel energy functional minimization model for speckle noise removal. Springer.

4. Cheng, Z., Han, P., Han, B., \& Sun, J. (n.d.). Classification of Polarimetric SAR Image based on Improved Fuzzy Clustering.

5. HIMABINDU, $K$., and $S$. JYOTHI."PROTEIN STRUCTURE COMPARISON USING FUZZY IMAGE PROCESSING. "International Journal of Computer Science Engineering and Information Technology Research (IJCSEITR) 6. 5, Oct 2016, 5358.

6. Huang, B., Mu, Y., Pan, Z., Bai, L., Yang, H., Access, J.-I., \& 2019, u. (n.d.). Speckle noise removal convex method using higherorder curvature variation. ieeexplore.ieee.org.

7. Khare, S., \& Kaushik, P. (2019, 7 10). Gradient nuclear norm minimization-based image filter. Modern Physics Letters B, 33(19).

8. Khare, S., B, P.-M., \& 2019, u. (n.d.). Gradient nuclear norm minimization-based image filter. World Scientific.

9. Liu, T., Yang, Z., Yang, J., Topics, G.-I., \& 2019, u. (n.d.). CFAR Ship Detection Methods Using Compact Polarimetric SAR in a K-Wishart Distribution. ieeexplore.ieee.org. 
10. Liu, X., Lu, J., Shen, L., Xu, C., \& Xu, Y. (2020, 2 18). Multiplicative Noise Removal: Nonlocal Low-Rank Model and Its Proximal Alternating Reweighted Minimization Algorithm.

11. Liu, X., Lu, J., Shen, L., Xu, C., \& Xu, Y. (n.d.). Multiplicative Noise Removal: Nonlocal Low-Rank Model and Its Proximal Alternating Reweighted Minimization Algorithm *.

12. Mahapatruni, N., Gopi Krishna, P., Gandi, R. K., \& Kuriti, R. (2020). IMPLEMENTATION STRATEGY OF MEAN AND FUZZY FILTERS IN REMOVING GAUSSIAN NOISE FROM IMAGES. Journal of Critical Reviews, 7, 566-570. Retrieved from http://www.jcreview.com/index.php?mno=23027\#cite.

13. Misra, A., Jones, C., \& Lim, H. (n.d.). Edge Enhancing Accelerated Diffusion Model for Speckle Denoising in Medical Imagery. Itm-conferences.org.

14. Rajalakshmi, M., and P. Subashini. "REMOVAL OF NOISE IN THE CHILI PEPPER IMAGES USING WEIGHTED 4CONNECTED MEDIAN FILTER." International Journal of Computer Science Engineering and Information Technology Research (IJCSETTR) (2013): 109118.

15. Shaik Mahaboob Basha, \& Jinaga, B. (2020). Robust Denoising Technique for Ultrasound Images Based on Weighted Nuclear Norm Minimization. Advances in Intelligent Systems and Computing. 1087, pp. 741-749. Springer.

16. SHARMA, NEHA, and YOGESH KUMAR."A SYSTEMATIC APPROACH FOR DESPECKLING OF MEDICAL ULTRASOUND IMAGES. "International Journal of Computer Science Engineering and Information Technology Research (IJCSEITR) 3.4, Oct 2013, 912.

17. Thankachan, R., Sethunadh, R., \& Ameer, P. (2019). Spatially adaptive polarimetric image despeckling using bandelet transform. European Journal of Remote Sensing.

18. Wang, R., He, N., Wang, Y., \& Lu, K. (2020, 3 1). Adaptively weighted nonlocal means and TV minimization for speckle reduction in SAR images. Multimedia Tools and Applications, 79(11-12), 76337647.

19. Wang, R., He, N., Wang, Y., Applications, K.-M., \& 2020, u. (n.d.). Adaptively weighted nonlocal means and TV minimization for speckle reduction in SAR images. Springer.

20. Wang, W., Xiang, D., Ban, Y., Zhang, J., \& Wan, J. (2019). Super pixel-Based Segmentation of Polarimetric SAR Images through Two-Stage Merging. mdpi.com.

21. WALI, AS, and AMIT TYAGI."NEURAL NETWORK MODEL SELECTION FOR DYNAMIC STRAIN MEASUREMENT USING FREQUENCY-DOMAIN PARAMETERS OF FIBER OPTIC SENSOR. "International Journal of Mechanical and Production Engineering Research and Development (IJMPERD) 9.5, Oct 2019, 319-332.

22. Zhang, W., Hu, B., Water, G., \& 2020, u. (n.d.). Automatic Surface Water Mapping Using Polarimetric SAR Data for LongTerm Change Detection. mdpi.com. 


\section{AUTHOR'S PROFILE}

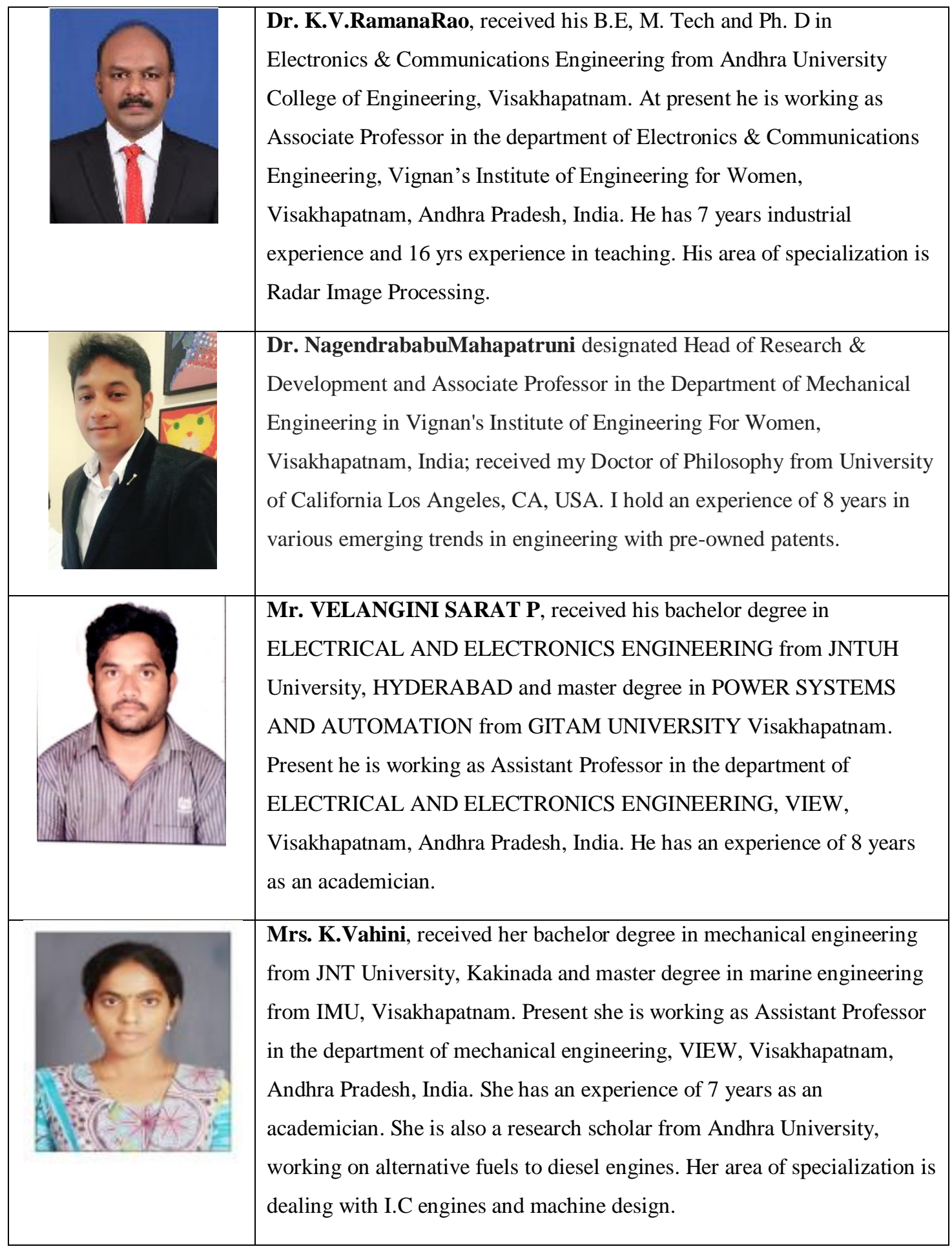

